\title{
SPECTROSCOPIC FEATURES OF CAFFEIC ACID: THEORETICAL STUDY
}

\author{
Jelena Tošović \\ Department of Chemistry, Faculty of Science, University of Kragujevac, \\ 12 Radoja Domanovića, 34000 Kragujevac, Serbia \\ E-mail: jelena.tosovic@kg.ac.rs
}

(Received March 21, 2017; Accepted March 25, 2017)

\begin{abstract}
In order to investigate spectroscopic features of caffeic acid (CA), the IR, Raman, ${ }^{13} \mathrm{C}-\mathrm{NMR},{ }^{1} \mathrm{H}-\mathrm{NMR}$, and UV spectra of this compound were simulated. For this purpose the B3LYP-D3/6-311+G(d,p) theoretical model was used in combination with CPCM solvation model. Very good agreement between all experimental and simulated spectra was achieved. This result indicates that B3LYP-D3 can be a method of choice in studies that refer to spectroscopic investigations. Spectroscopic features of CA are very similar to those of chlorogenic acid (5-O-caffeoylquinic acid) (MARKOVIĆ, TOŠOVIĆ and DIMITRIĆ MARKOVIĆ, 2016), pointing out the significance of caffeic moiety.
\end{abstract}

Key words: caffeic acid, vibrational spectra, NMR spectra, UV spectrum, DFT calculations

\section{INTRODUCTION}

Phenolic acids are derivatives of cinnamic and benzoic acids. These compounds are widely spread in food. It is well known that they have an impact on flavour, stability, nutritional value, colour and other food properties (VAUGHAN and GEISSLER, 2009). It has been recognized that phenolic acids possess numerous biological roles due to their ability to scavenge free radicals, inhibit lipoxygenase, and chelate metals (DECKER, 1997; JAYASENA et al., 2013). In addition, the consumption of phenolic acids through vegetables and fruits has a beneficial effect on health (PULIDO et al., 2000).

Phenolic acids can be divided into two major groups: hydroxybenzoic acids and hydroxycinnamic acids. Hydroxybenzoic acids include p-hydroxybenzoic, syringic, protocatechuic, gallic, and vanilic acids. Hydroxycinnamic acids are characterized with C6C3 skeleton and include sinapic, coumaric, ferulic and caffeic acids. In nature, only small number of these compounds exists in free form, whereas the conjugates of polysaccharides, sugars, or organic acids are more frequently observed. One of the most representative dietary hydroxycinnamic acids is caffeic acid (3,4-dihydroxycinnamic acid, CA, Fig. 1). 


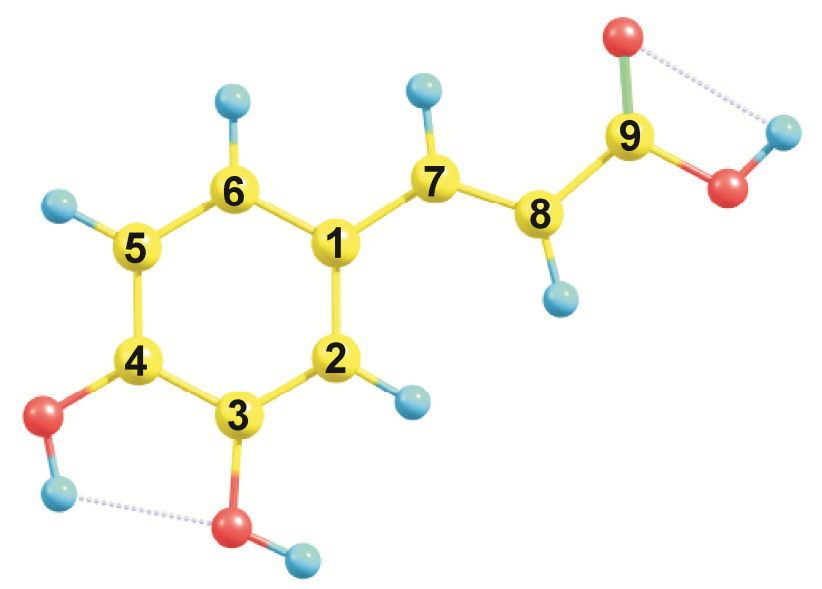

Figure 1. Structure of caffeic acid with the atom labelling scheme.

CA can be found in citrus fruits, apples, juices, bran of cereal grains, and especially in coffee. In food, it usually exists in a form of ester with quinic acid as chlorogenic acid (5-Ocaffeoylquinic acid). As a precursor of many biologically active polyphenols in phenylpropanoid metabolism, CA plays a very important role in the defence mechanism of plants against predation and infection (FAULDS and WILLIAMSON, 1999; BUCHANAN et al., 2000). It can also protect nascent leaves from UV-B radiation (GouLD et al., 2000). The bioassay experiments have revealed that CA has an impact on the inhibition of the growth of insects, fungi, and bacteria (BAGHESTANI et al., 1999; BOSTOCK et al., 1999; REINDERS et al., 2001). In addition, the level of this compound in plant species is related to allelopathic potential and pest resistance (SNOOK et al., 1994; BAGHESTANI et al., 1999). The beneficial effect of CA in vivo has also been reported (FukUMOTO and MAZZA, 2000; GULCIN, 2006). Numerous biological and pharmacological properties of this compound, such as antiinflammatory (JOYEUX et al., 1995), anticancerogenic (CHALLIS and BARTLETT, 1975), antiviral (FRANK et al., 1989) and ability to completely block the production of reactive oxygen species (ROS) (SUDINA et al., 1993) have been discovered. In addition, the daily consumption of coffee lowers the risk of cardiovascular diseases and diabetes (JOKURA et al., 2015).

CA exists in a form of yellow solid and the exact structure of this compound is known from the X-ray experiment (GARCíA-GRANDA et al., 1987). There are a few experimental studies devoted to vibrational, NMR and UV spectra of CA (WANG et al., 2004; EUN et al., 2010; ŚWISŁOCKA, 2013). The goal of the present study is to investigate spectroscopic properties of CA by theoretical means. An additional aim is to examine the performance of the B3LYP-D3 method in investigations devoted to the spectroscopic features.

\section{COMPUTATIONAL DETAILS}

Gaussian 09 program package was employed for all quantum chemical calculations (FRISCH et al., 2013). The B3LYP-D3/6-311+G(d,p) theoretical model was applied for the optimization and frequency calculations in the gas-phase and solution. To mimic experimental conditions the calculations were performed in methanol solution (dielectric constant $\varepsilon=$ 32.6130). For this purpose, the CPCM polarizable continuum solvation model was selected. The gas-phase geometry of CA was used to simulate the IR and Raman spectra, whereas the optimized geometry in methanol was used to predict the NMR and UV spectra. The vibrational modes of CA were assigned on the basis of the potential energy distribution (PED) 
analysis (MUNOS et al., 1970) using the FCART 7.0 software (LEGLER et al., 2015). The ${ }^{13} \mathrm{C}$ NMR and ${ }^{1} \mathrm{H}$ properties of $\mathbf{C A}$ were predicted by means of gauge independent atomic orbital (GIAO) method. The time dependent density functional theory (TDDFT) calculations were applied for the simulation of the UV spectrum of this compound. To provide an informative description of electronic transitions, the B3LYP-D3 ground state geometry was used to perform the NBO (Natural Bond Orbital) analysis, and construct the NLMO clusters (WEINHOLD and LANDIS, 2012). The NLMO clusters were created using the strategy applied in our recent papers (MARKOVIĆ and ToŠOVIĆ, 2015; ToŠOVIĆ and MARKOVIĆ, 2017).

\section{RESULTS AND DISCUSION}

\section{Vibrational spectra of $C A$}

The vibrational spectra of CA consist of in total 57 vibrational modes. Due to the fact that the vibrational scaling factor for the B3LYP-D3/6-311+G(d,p) model has not been reported, it was determined on the basis of the experimental data for the IR spectrum using the least squares method, and it amounts to 0.922.The calculated values of the wavenumbers were scaled and assigned to the corresponding experimental values taken from literature (ŚwISŁOCKA, 2013). The most important vibrational modes of CA and corresponding PED values are presented in Table 1.

Table 1. Characteristic experimental (ŚWISŁOCKA, 2013) and calculated wavenumbers (v) in the vibrational spectra of caffeic acid along with the assignments and intensities of the normal modes.

\begin{tabular}{ccccc}
\hline Mode assignment & VIR $_{\text {IR }} \begin{array}{c}\text { VRaman } \\
\text { Exp./ cm }\end{array}$ & $\begin{array}{c}\text { B3LYP-D3 } \\
\text { Calc./ cm-1 }\end{array}$ & PED (\%) \\
\hline H stretching (b ${ }^{*}$ ) & $3431 \mathrm{~s}^{\#}$ & & 3550 & $v_{\mathrm{OH}}(99)$ \\
OH stretching (b) & $3231 \mathrm{~s}$ & & 3493 & $v_{\mathrm{OH}}(99)$ \\
OH stretching (a) & & & 3477 & $v_{\mathrm{OH}}(100)$ \\
CH stretching (b) & $3057 \mathrm{w}$ & & 2950 & $v_{\mathrm{CH}}(98)$ \\
CH stretching (a) & $3026 \mathrm{w}$ & & 2944 & $v_{\mathrm{CH}}(98)$ \\
CH stretching (b) & $2988 \mathrm{w}$ & & 2920 & $v_{\mathrm{CH}}(98)$ \\
CH stretching (a) & $2926 \mathrm{w}$ & & 2903 & $v_{\mathrm{CH}}(98)$ \\
CO stretching (a) & $1645 \mathrm{vs}$ & $1642 \mathrm{~s}$ & 1638 & $v_{\mathrm{CO}}(78)+v_{\mathrm{CC}}(3)+$ \\
& & & 1547 & $\delta_{\mathrm{COH}}(5)+\delta_{\mathrm{OCC}}(3)+\delta_{\mathrm{HCC}}(5)+$ \\
CC stretching (b,a) & $1620 \mathrm{vs}$ & $1615 \mathrm{vs}$ & $\delta_{\mathrm{CCH}}(4)$ \\
CC stretching (b,a) & $1524 \mathrm{~m}$ & $1525 \mathrm{vw}$ & 1515 & $v_{\mathrm{CC}}(49)+v_{\mathrm{OC}}(8)$ \\
CC stretching (b,a) & $1450 \mathrm{vs}$ & $1453 \mathrm{~m}$ & 1435 & $v_{\mathrm{CC}}(8)+\delta_{\mathrm{CCH}}(10)+$ \\
\end{tabular}

" $\mathrm{b}$ and a stand for benzene moiety and acyclic chain

${ }^{\#} \mathrm{vs}$ - very strong, s - strong, $\mathrm{m}$ - medium, w - weak, vw - very weak

The quality of the linear correlation between the experimental and calculated wavenumbers was evaluated by means of three descriptors: the correlation coefficient $(\mathrm{R})$, 
average absolute error (AAE), and average relative error (ARE). The $\mathrm{R}$ value for the IR and Raman spectra is identical and amounts to 0.998 (Figs. 2 and 3). The AAE and ARE values for the IR spectrum amount to $36.1 \mathrm{~cm}^{-1}$ and $2.5 \%$, whereas for the Raman spectrum AAE and ARE are equal to $16.2 \mathrm{~cm}^{-1}$ and $1.8 \%$. Relatively higher values for AAE and ARE for IR relative to Raman spectrum originate from notable differences between the values for experimental and calculated $\mathrm{OH}$ starching modes. This deviation can be explained by the fact that simulated vibrational spectra refer to an isolated molecule of $\mathbf{C A}$, implying that intermolecular interactions, including hydrogen bonds, are neglected. These interactions, particularly intermolecular hydrogen bonds, are of great importance in a real solid-state CA.

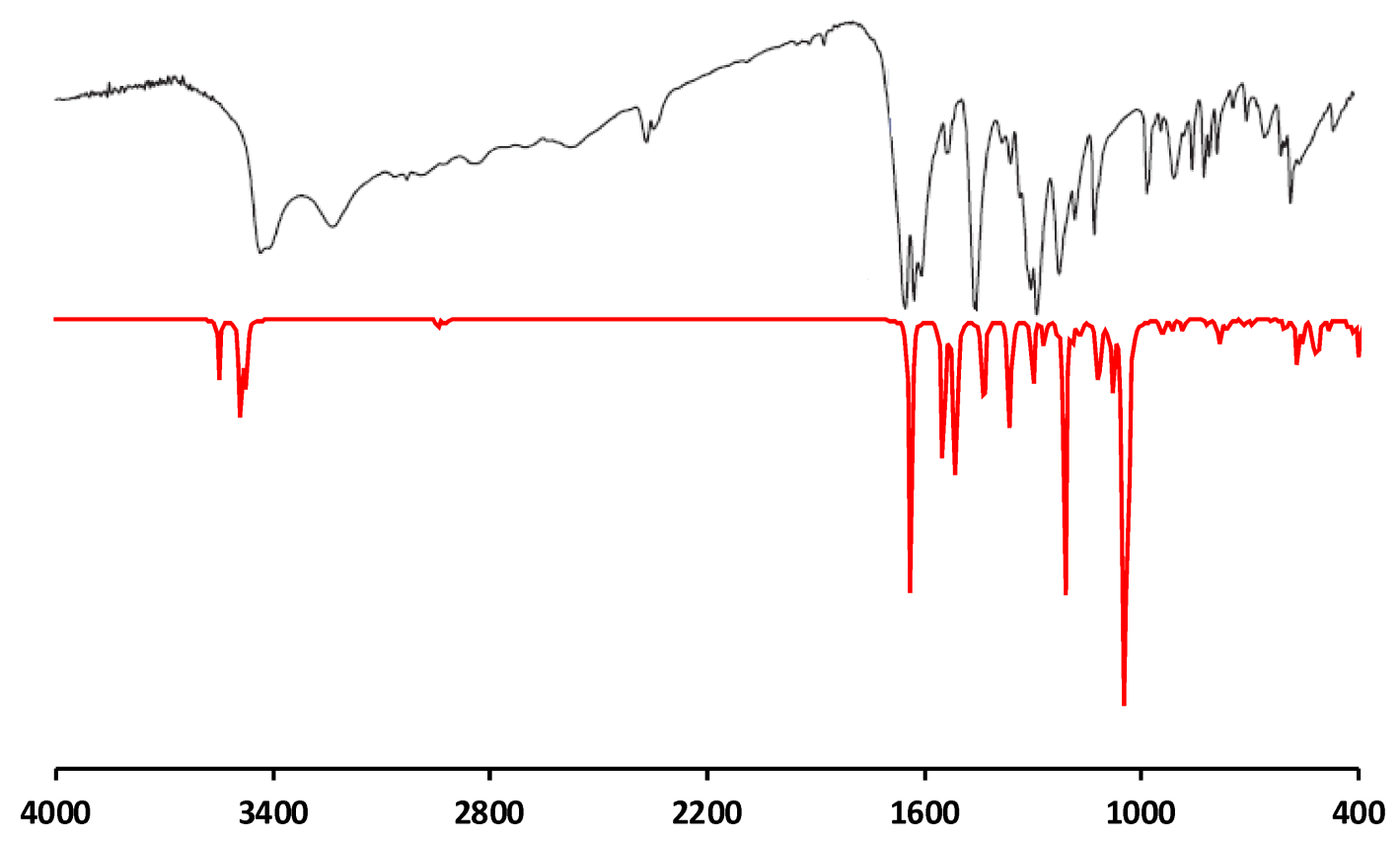

Figure 2. Experimental (ŚwISŁOCKA, 2013) (up) and calculated (bottom) IR spectrum of caffeic acid.

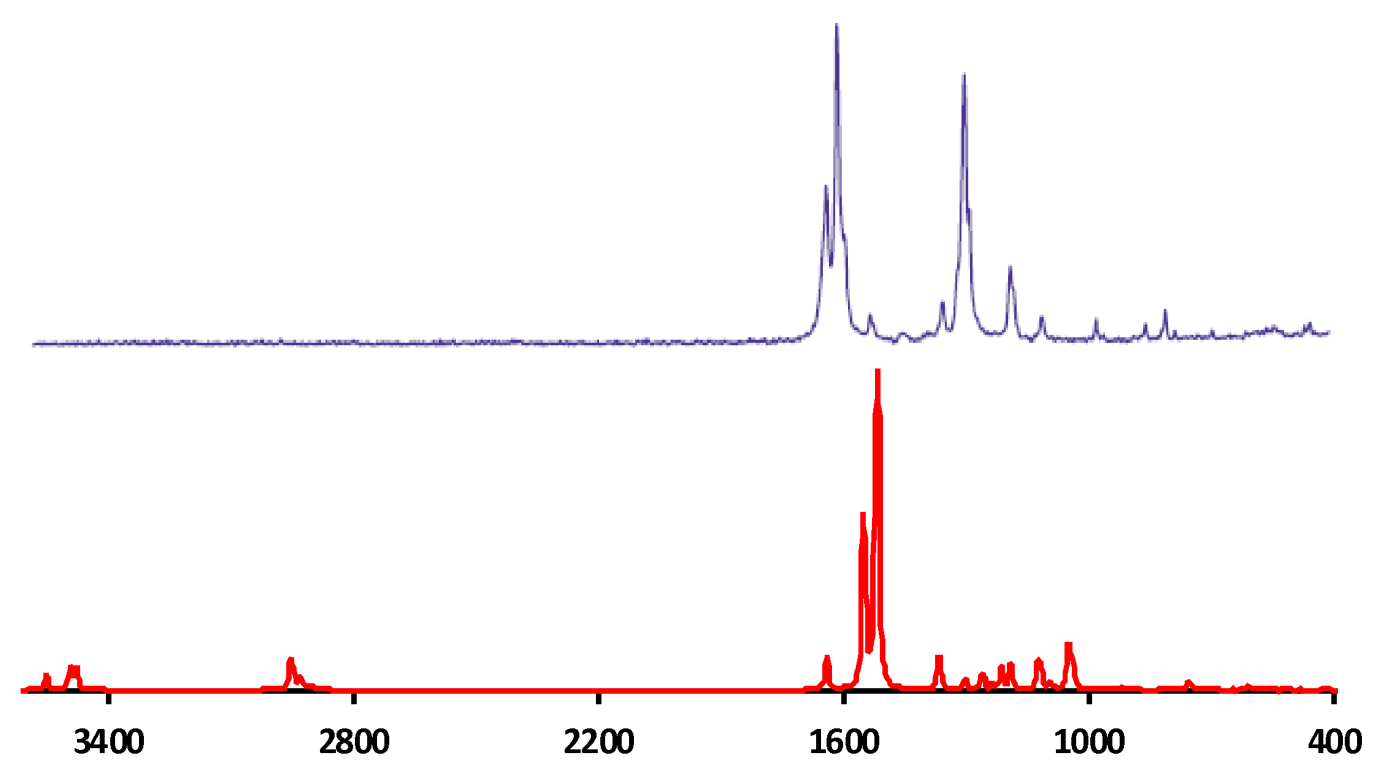

Figure 3. Experimental (ŚwISŁOCKA, 2013) (up) and calculated (bottom) Raman spectrum of caffeic acid. 
Very massive and intense overlapping bands in the IR spectrum, which appear in the region of 4000-2600 $\mathrm{cm}^{-1}$, were assigned to the different modes of the $\mathrm{OH}$ vibrations. The high frequency region is also characterized by the week $\mathrm{CH}$ stretching modes of benzene moiety and acyclic chain. The vibrational contributions to the normal stretching modes (PED values in Table 1) in the $3431-2926 \mathrm{~cm}^{-1}$ region are assigned almost solely to the $\mathrm{OH}$ and $\mathrm{CH}$ stretching modes themselves $(\sim 100 \%)$ while the rest of the modes are presented as the combination of various contributions.

The bands of the very strong and strong intensities at 1645 (IR) and $1642 \mathrm{~cm}^{-1}$ (R) were assigned to the $\mathrm{CO}$ stretching modes of the carboxyl group. In addition, the bands of the very strong and medium intensities in the IR spectrum at 1620,1524 , and $1450 \mathrm{~cm}^{-1}$, and very strong, very weak, and medium intensities in the Raman spectrum at 1615, 1525, and 1453 $\mathrm{cm}^{-1}$ were mostly assigned to the CC stretching modes of both benzene moiety and acyclic chain.

\section{NMR spectra of CA}

The chemical shifts for the carbon and hydrogen atoms of CA relative to TMS were calculated. Since the chemical shifts were systematically overestimated, their values were scaled, and these scaled chemical shifts were further used. The scaling factors were determined by means of the least squares method, and their values for the ${ }^{13} \mathrm{C}$ - and ${ }^{1} \mathrm{H}-\mathrm{NMR}$ spectra are as follows: 0.955 and 0.946. The obtained results are collected in Table 2.

The correlation coefficients for ${ }^{13} \mathrm{C}-\mathrm{NMR}$ and ${ }^{1} \mathrm{H}-\mathrm{NMR}$ are equal to 0.980 and 0.990 , whereas the AAE values are equal to 3.17 and $0.05 \mathrm{ppm}$, respectively. A careful inspection of Table 2 reveals that the calculated chemical shifts for $\mathrm{C} 2, \mathrm{C} 6, \mathrm{C} 7$, and $\mathrm{C} 8$ carbons deviate from the experimental values. This discrepancy is a consequence of free rotation around the $\mathrm{C} 1-\mathrm{C} 7$ and $\mathrm{C} 8-\mathrm{C} 9$ single bonds. The agreement between the experimental and simulated ${ }^{1} \mathrm{H}-$ NMR spectra is very good.

Table 2. Experimental (EUN et al., 2010) and calculated chemical shifts (ppm) for ${ }^{13} \mathrm{C}-$ and ${ }^{1} \mathrm{H}-\mathrm{NMR}$ spectra of caffeic acid in methanol.

\begin{tabular}{ccc|cc}
\hline & \multicolumn{2}{c|}{${ }^{\mathbf{1 3}} \mathbf{C}$} & \multicolumn{2}{c}{${ }^{\mathbf{1}} \mathbf{H}$} \\
Position & Calc. & Exp. & Calc. & Exp. \\
\hline $\mathbf{1}$ & 127.9 & 127.8 & & \\
$\mathbf{2}$ & 110.8 & 115.6 & 7.11 & 7.02 \\
\hline $\mathbf{3}$ & 144.8 & 146.8 & & \\
$\mathbf{4}$ & 151.0 & 149.5 & & \\
$\mathbf{5}$ & 116.1 & 116.5 & 6.78 & 6.77 \\
\hline $\mathbf{6}$ & 130.0 & 122.8 & 6.84 & 6.92 \\
\hline $\mathbf{7}$ & 152.2 & 147.0 & 7.55 & 7.52 \\
\hline $\mathbf{8}$ & 110.4 & 115.1 & 6.14 & 6.20 \\
\hline $\mathbf{9}$ & 168.5 & 171.1 & & \\
\hline
\end{tabular}




\section{UV spectrum of $C A$}

The experimental UV spectrum of CA in methanol shows four absorption bands at 327, 295, 243, and $217 \mathrm{~nm}$ (Table 3) (WANG et al., 2004). The calculated UV spectrum was obtained using the TDDFT method. The results of TDDFT approach, including wavelengths $\left(\lambda_{\max }\right)$, oscillator strengths $(f)$, and orbital contribution coefficients, are shown in Table 3 . A comparison between the experimental and calculated $\lambda_{\max }$ values reveals an excellent performance of the B3LYP-D3 level of theory.

A deeper analysis of the UV spectrum will be based on the combined results from the TDDFT theory, which provides a prediction of energy levels (Table 3), and NBO theory, which provides NLMO clusters (Fig. 4). NLMO cluster should be understood as a part of a molecule characterized with distinguished electron density which is utilized to identify the parts of molecules involved in electronic transitions.

Table 3. Results of the TDDFT/B3LYP-D3 calculations. $\lambda_{\max }$ denotes experimental (WANG et al., 2004) and calculated transition wavelengths, whereas $f$ stands for oscillator strengths.

Orbital description points out pairs of MOs involved in electronic transitions.

\begin{tabular}{cccc}
\hline $\begin{array}{c}\lambda_{\max } \\
\text { Calc. }(\mathbf{n m})\end{array}$ & Orbital description & $\boldsymbol{f}$ & $\begin{array}{c}\lambda_{\max } \\
\text { Exp. }(\mathbf{n m})\end{array}$ \\
\hline 333 & HOMO $\rightarrow$ LUMO (69\%) & 0.64 & 327 \\
294 & HOMO-1 $\rightarrow$ LUMO (69\%) & 0.13 & 295 \\
246 & HOMO $\rightarrow$ LUMO+1 (63\%) & 0.07 & 243 \\
222 & HOMO-3 $\rightarrow$ LUMO (64\%) & 0.19 & 217 \\
\hline
\end{tabular}

In Fig. 4 the relevant NLMO clusters for $\mathbf{C A}$ are depicted. The most intensive band at the smallest excitation energy results from the HOMO $\rightarrow$ LUMO electronic transitions at 327 nm (exp.) (Table 3). When molecular moieties are considered (Fig. 4) it turns out that this transition can be attributed to $\pi \rightarrow \pi^{*}$ transition from the caffeic moiety to the $\mathrm{C}=\mathrm{O}$ carbonyl group. Due to existence of the shearing region between the HOMO and LUMO clusters, this transition involves a favourable intramolecular charge transfer. In addition, small HOMO LUMO energy gap makes this electronic transition most favourable, which is reflected through the largest oscillator strength. An $n \rightarrow \pi^{*}$ electronic transition from carbonyl group ( $p$ orbital) to carbonyl group ( $\pi^{*}$ orbital) is responsible for the absorption band at $295 \mathrm{~nm}$ (HOMO-1 $\rightarrow$ LUMO). This band, as well as all other transitions, is characterized with smaller $f$ value because of larger energy separation. The next bend at $243 \mathrm{~nm}(\mathrm{HOMO} \rightarrow \mathrm{LUMO}+1)$ results from transition from caffeic moiety ( $\pi$ orbital) to the aromatic ring ( $\pi^{*}$ orbital). The last band at $217 \mathrm{~nm}$ (HOMO-3 $\rightarrow$ LUMO) originates from $\pi \rightarrow \pi^{*}$ electronic transition from carbonyl group ( $\pi$ orbital) to carbonyl group ( $\pi^{*}$ orbital). Fig. 4 shows that all electronic transitions are characterized with small spatial separations, whereas energetic separations are proportional to the excitation energies. 


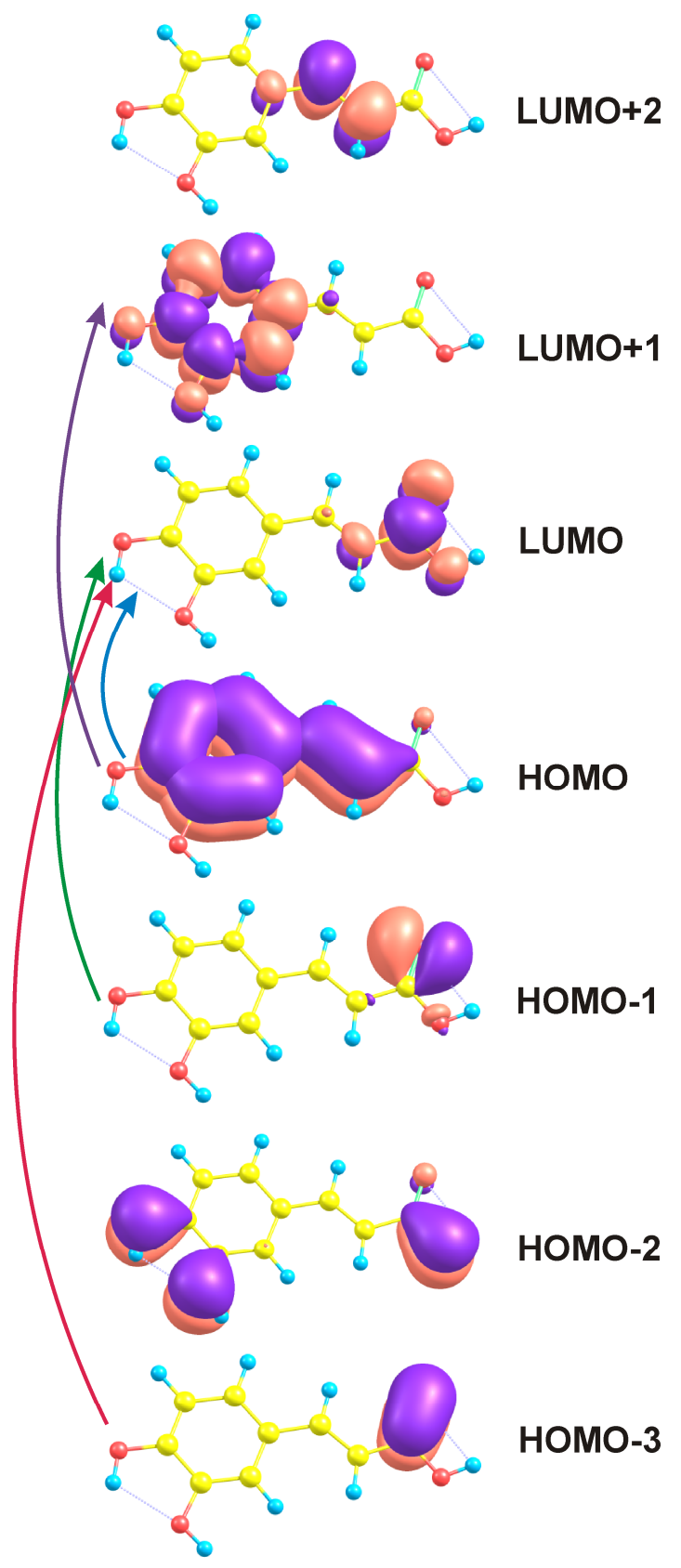

Figure 4. The NLMO clusters of caffeic acid. The arrows connect molecular moieties involved in electronic transitions.

\section{CONCLUSION}

The B3LYP-D3 method provides a very good agreement between all calculated and experimental spectra of CA. The result of the present study agrees with our previous results for the simulation of the spectra for different compounds obtained with the same method (TOŠOVIĆ and MARKOVIĆ, 2017) and indicates that the B3LYP-D3 method is a good choice for investigations of spectroscopic features.

The most important source of slight disagreement between the experimental and calculated band positions in vibrational spectra can be assigned to the fact that the 
calculations consider an isolated molecule and ignore intermolecular interactions which are of great importance in a real solid-state CA.

Some discrepancy in the values for chemical shifts is a consequence of rotation around single bonds, and is most evident on the $\mathrm{C} 2$ and $\mathrm{C} 6$ atoms (rotation of the aromatic ring around the $\mathrm{C} 1-\mathrm{C} 7$ single bond), as well as on $\mathrm{C} 7$ and $\mathrm{C} 8$ atoms (rotation around the $\mathrm{C} 8-\mathrm{C} 9$ single bond).

The NLMO clusters were constructed in order to provide an informative description of the UV spectrum. The absorption bands result from the $\pi \rightarrow \pi^{*}$ or $n \rightarrow \pi^{*}$ electronic transitions. It was found that all electronic transitions are characterized with small spatial separations, whereas energetic separations are proportional to the excitation energies.

It should be pointed out that both experimental and calculated spectroscopic properties, obtained in present work for $\mathbf{C A}$, and those obtained in our recent paper for chlorogenic acid (MARKOVIĆ et al., 2016), are mutually very similar. This finding indicates that caffeic moiety is the most responsible for spectroscopic properties of chlorogenic acid.

\section{Acknowledgments}

This work was supported by the Ministry of Science and Technological Development of the Republic of Serbia (Grant no 172016).

\section{References:}

[1] Baghestani, A., Lemieux, C., Leroux, G.D., Baziramkenga, R., Simard, R.R. (1999): Determination of allelochemicals in spring cereal cultivars of different competitiveness. Weed Sci. 47: 498-504.

[2] Bostock, R.M., Wilcox, S.M., Wang, G., Adaskaveg, J.E. (1999): Suppression of Monilinia fructicola cutinase production by peach fruit surface phenolic acids. Physiol. Mol. Plant Pathol. 54: 37-50.

[3] Buchanan, B.B., Gruissem, W., Jones, R.L. (2000): Biochemistry and Molecular Biology of Plants, Rockville, MD: American Society of Plant Physiology.

[4] Challis, B.C., Bartlett, C.D. (1975): Possible cocarcinogenic effects of coffee constituents. Nature 254: 532-533.

[5] DECKER, E.A., (1997): Phenolics: prooxidants or antioxidants? Nutr. Rev. 55: 396-398.

[6] Eun, J.L., KIM, J.S., KIM, H.P., LEE, J.-H., KANG, S.S. (2010): Phenolic constituents from the flower buds of Lonicera japonica and their 5-lipoxygenase inhibitory activities. Food Chem.120: 134-139.

[7] Faulds, C.B., Williamson, G. (1999): The role of hydroxycinnamates in the plant cell wall. J. Sci. Food Agric. 79: 393-395.

[8] Frank, H., ThIEL, D., MACleOD, J. (1989): Mass spectrometric detection of cross-linked fatty acids formed during radical-induced lesion of lipid membranes. Biochem. J. 260: 873-878. 
[9] Frisch, M.J., Trucks, G.W., Schlegel, H.B., Scuseria, G.E., Robb, M.A., Cheeseman, J.R., Scalmani, G., Barone, V., Mennucci, B., Petersson, G.A. et al. (2013): Gaussian 09, Revision D.1. Wallingford CT: Gaussian Inc.

[10] Fukumoto, L.R., MAZZA, G. (2000): Assessing antioxidant and prooxidant activities of phenolic compounds. J. Agric. Food Chem. 48: 3597-3604.

[11] García-Granda, S., Beurskens, G., Beurskens, P.T., Krishna, T.S.R. (1987): Structure of 3,4-dihydroxy-trans-cinnamic acid (caffeic acid) and its lack of solid-state topochemical reactivity. Acta Crystallogr. C C43: 683-685.

[12] Gould, K.S., Markham, K.R., Smith, R.H., Goris, J.J. (2000): Functional role of anthocyaninis in the leaves of Quintinia serrata A. Cunn. J. Exp. Bot. 51: 1107-1115.

[13] Gulcin, I. (2006): Antioxidant activity of caffeic acid (3,4-dihydroxycinnamic acid). Toxicology 217: 213-220.

[14] Jayasena, T., Poljak, A., Smythe, G., Braidy, N., Münch, G., Sachdev, P. (2013): The role of polyphenols in the modulation of sirtuins and other pathways involved in Alzheimer's disease. Ageing Res. Rev. 12: 867-883.

[15] Jokura, H., Watanabe, I., Umeda, M., Hase, T., Shimotoyodome, A. (2015): Coffee polyphenol consumption improves postprandial hyperglycemia associated with impaired vascular endothelial function in healthy male adults. Nutr. Res.35: 873-881.

[16] Joyeux, M., Lobstein, A., Anton, R., Mortier, F. (1995): Comparative antilipoperoxidant, antinecrotic and scavenging properties of terpenes and biflavones from Ginkgo and some flavonoids. Planta Med. 61: 126-129.

[17] Legler, C.R., Brown, N.R., Dunbar, R.A., Harness, M.D., Nguyen, K., Oyewole, O., Collier, W.B. (2015): Scaled quantum mechanical scale factors for vibrational calculations using alternate polarized and augmented basis sets with the B3LYP density functional calculation model. Spectrochim. Acta A 145: 15-24.

[18] MARKović, S., ToŠOviĆ, J. (2015): Application of time-dependent density functional and natural bond orbital theories to the UV-vis absorption spectra of some phenolic compounds. J. Phys. Chem. A 119: 9352-9362.

[19] Marković, S., ToŠOvić, J., Dimitrić MARKović, J.M. (2016): Synergic application of spectroscopic and theoretical methods to the chlorogenic acid structure elucidation, Spectrochim. Acta A 164: 67-75.

[20] Munos, R.A., Panchenko, Y.N., Koptev, G.S., Stepanov, N.F. (1970): Program for calculating distribution of potential energy in internal coordinates. J. Appl. Spectrosc.12: 428-429.

[21] Pulido, R., Bravo, L., Saura-Calixto, F. (2000): Antioxidant activity of dietary polyphenols as determined by a modified ferric reducing/ antioxidant power assay. $J$. Agric. Food Chem. 48: 3396-3402.

[22] Reinders, R.D., Biesterveld, S., BiJKer, P.G.H. (2001): Survival of Escherichia coli O157:H7 ATCC 43895 in a model apple juicemedium with different concentrations of proline and caffeic acid. Appl. Environ. Microbiol. 67: 2863-2866.

[23] SNOOK, M.E., DATA, E.S., KAYS, S.J. (1994): Characterization and quantitation of hexadecyl, octadecyl and eicosyl esters of $p$-coumaric acid in the vine and root latex of sweetpotato [Ipomoea batatas (L.) lam.]. J. Agric. Food Chem.42: 2589-2595. 
[24] Sudina, G.F., Mirzoeva, O.K., Pushkareva, M.A., Korshunova, G.A., Sumbatyan, N.V., VARFolomeEv, S.D. (1993): Caffeic acid phenethyl ester as a lipoxygenase inhibitor with antioxidant properties. FEBS Lett. 329: 21-24.

[25] ŚWISŁOCKA, R. (2013): Spectroscopic (FT-IR, FT-Raman, UV absorption, ${ }^{1} \mathrm{H}$ and ${ }^{13} \mathrm{C}$ NMR) and theoretical (in B3LYP/6-311++G** level) studies on alkali metal salts of caffeic acid, Spectrochim. Acta A 100: 21-30.

[26] ToŠOviĆ, J., MARKOVIĆ, S. (2017): Reproduction and interpretation of the UV-vis spectra of some flavonoids. Chem. Pap.71: 543-552.

[27] Vaughan, J.G., Geissler, C.A. (2009): The New Oxford Book of Food Plants. New York: Oxford University Press.

[28] Wang, H., Provan, G. J., Helliwell, (2004): K. Determination of rosmarinic acid and caffeic acid in aromatic herbs by HPLC, Food Chem. 87: 307-311.

[29] WeINHOLD, F., LANDIS, C.R. (2012): Discovering Chemistry with Natural Bond Orbitals. Hoboken: John Wiley \& Sons, Inc. 